\title{
Reflection second harmonic generation on a $z$-cut congruent lithium niobate crystal
}

\author{
T. J. Sono*, J. G. Scott, C. L. Sones, C. E. Valdivia, S. Mailis and R. W. Eason \\ Optoelectronics Research Centre, University of Southampton \\ J. G. Frey \\ School of Chemistry, University of Southampton \\ L. Danos \\ School of Engineering Sciences, University of Southampton \\ Southampton, SO17 1BJ, UK
}

Reflection second harmonic generation experiments were performed on $z$-cut congruent lithium niobate crystals $\left(\mathrm{LiNbO}_{3}\right)$ to reveal the interfacial layer symmetry as the crystal is rotated around the $z$-axis. To suppress the bulk contribution, the fundamental wavelength was selected to be $532 \mathrm{~nm}$ resulting in second harmonic generation at a wavelength within the absorption region of the crystal. The polarity of the direction of the $y$-axis was determined from second harmonic generation data and used to show that this direction also inverts during domain inversion.

PACS numbers(s): 42.65.Ky, 42.70.Mp, 77.84.Dy

Key words: $S H G$, lithium niobate, $3 m$, crystalline symmetry, axis inversion 


\section{INTRODUCTION}

Within the electric dipole approximation, second harmonic generation (SHG) is forbidden in centrosymmetric media ${ }^{1}$, however, at the surface it becomes allowed due to the relaxation of the inversion symmetry. Reflection SHG (RSHG) has been one of the major tools for selective surface studies for a wide range of material interfaces and surfaces. ${ }^{2}$ Surface studies such as symmetry determination using SHG are normally achieved, for example, by measuring SHG signals with a well defined input and output beam geometry, while rotating the sample around the axis normal to the surface. This is feasible since the irreducible components of the second order susceptibility $\left(\chi^{(2)}\right)$ tensor reflect the symmetry of the medium and hence of the surface. In general, the structural symmetry of the surface and that of the bulk are not the same due to the termination and discontinuity of the bulk symmetrical properties at the interface. Better understanding of electronic structure of the surface is of great importance since this determines the behaviour of the surface.

For non-centrosymmetric materials, the surface selectivity by SHG is limited due to the inherent contribution from the bulk. It has been shown that with suitable selection of the input and output beam polarization and the azimuthal angles, the SHG contribution from the bulk and the surface can be separated. ${ }^{3}$ This method is however not applicable for the determination of the symmetry of the interfacial layer where the azimuthal or the polarization angles must be varied. $\mathrm{LiNbO}_{3}$ exhibits high UV absorption hence the penetration of UV light is restricted to within a few hundred atomic layers in this material. Thus for $\mathrm{LiNbO}_{3}$, surface selectivity using RSHG can be achieved by selecting a fundamental beam in the visible range and hence a SHG beam in the UV from the top outer-most atomic layer. At 266 $\mathrm{nm}$, both ordinary and extraordinary polarized light can only propagate for a1/e distance of $\sim 15 \mathrm{~nm}^{4,5}$ Any SHG signal generated deeper than the interfacial layer will be absorbed by 
the bulk and will not contribute to the observed reflected signal. This layer is, however, thicker than what can be considered as the surface (few tenths of atomic layers) and therefore it is called an interfacial layer. The difference between this layer and the surface is that its optical properties are similar to that of the bulk while in general they differ from that of the surface. Thus the observed RSHG will include signal from both the surface and the interfacial layer. We show in this paper nterfacial layer symmetry studies on a $z$-cut congruent $\mathrm{LiNbO}_{3}$ crystal using the RSHG technique.

Below the Curie temperature, the combination of several properties of $\mathrm{LiNbO}_{3}$ such as ferroelectricity, high nonlinearity and high optical damage threshold has lead to the use of this crystal in many technological applications such as quasi phase-matching (QPM). With ferroelectric materials, QPM devices are made by periodic reversal of the spontaneous polarization $\left(\mathbf{P}_{\mathbf{s}}\right)$. For $\mathrm{LiNbO}_{3}, \mathbf{P}_{\mathbf{s}}$ is along the $z$-axis and hence its reversal implies the reversal of the $z$-axis $(+z \Leftrightarrow-z)$. In this paper we will also illustrate, as a special case, the use of RSHG to detect the reversal of the $y$-axis $(+y \Leftrightarrow-y)$ that accompanies the reversal of the spontaneous polarisation along the $z$-axis. Below, we outline firstly the relation between the second order polarization and hence SH signal to the symmetry of the crystal by considering the transformation of the non-vanishing components $\chi^{(2)}$ as the sample is rotated around the axis normal to the surface.

\section{THEORY}

Using a three layer model for the RSHG, the interfacial layer is taken as a middle layer with a finite thickness $(\boldsymbol{d})$ between the top medium (air in this case) and the bulk material. $\boldsymbol{d}$ is taken to be very small compared to the wavelength of the light used. ${ }^{6}$ Since in this paper the very short absorption length of $\mathrm{LiNbO}_{3}$ is used to limit the SHG bulk contribution, all $\chi^{(2)}$ tensor 
coefficients are complex and the Kleinman conjecture7 does not hold. To use the RSHG for interfacial layer symmetry determination, the components of the $\chi^{(2)}$ tensor within the crystal are transformed to the laboratory-fixed axes system and below we summarise this transformation for a material with a $3 \mathrm{~m}$ symmetry.

Let $(I, J, K)$ and $(i, j, k)$ denote the laboratory-fixed axes and the crystal-fixed axes respectively. Both axes systems are Cartesian and have principle axes given as $(X, Y, Z)$ for the laboratoryfixed axes and $(x, y, z)$ for the crystal-fixed axes where the $Z$ - axis and $z$-axis ( $c$-axis) remain parallel as shown by Figure 1. As the sample is rotated, the components of the interfacial layer second harmonic polarization are related to the components of the inducing fields within the medium by a second-order laboratory-defined $\operatorname{susceptibility~tensor}\left(\chi_{I J K}^{(2)}\right)$ given by

$$
P_{I}^{2 \omega}=\chi_{I J K}^{(2)} E_{J}^{(\omega)} E_{K}^{(\omega)}
$$

Where $\chi_{I J K}^{(2)}$ is related to the interfacial layer second-order susceptibility $\chi_{i j k}^{(2)}$ tensor within the crystal via $^{1}$

$$
\chi_{I J K}^{(2)}=R_{I i} R_{J j} R_{K k} \chi_{i j k}^{(2)}
$$

In equation (2), $R_{I i}$ is the element of the rotation matrix containing the directional cosines between the laboratory-fixed and crystal-fixed axes. For a $3 \mathrm{~m}$ symmetry, the components of the laboratory-fixed second harmonic polarization as a function of the azimuthal angle $(\varphi)$, deduced from equation (2) can be written as ${ }^{8-10}$ 


$$
\left[\begin{array}{c}
P_{x}^{2 \omega} \\
P_{y}^{2 \omega} \\
P_{z}^{2 \omega}
\end{array}\right]=\left[\begin{array}{cccccc}
\chi_{y y y} K_{1} & -\chi_{y y y} K_{1} & 0 & 0 & \chi_{x x z} & \chi_{y y y} K_{2} \\
\chi_{y y y} K_{2} & -\chi_{y y y} K_{2} & 0 & \chi_{x x z} & 0 & -\chi_{y y y} K_{1} \\
\chi_{z x x} & \chi_{z x x} & \chi_{z z z} & 0 & 0 & 0
\end{array}\right]\left[\begin{array}{c}
E_{x}^{2} \\
E_{y}^{2} \\
E_{z}^{2} \\
2 E_{y} E_{z} \\
2 E_{x} E_{z} \\
2 E_{x} E_{y}
\end{array}\right]
$$

where $K_{1}=\left(-3 \sin (\varphi) \cos ^{2}(\varphi)+\sin ^{3}(\varphi)\right)$ and $K_{2}=\left(3 \sin ^{2}(\varphi) \cos (\varphi)-\cos ^{3}(\varphi)\right)$.

As shown in Figure 1, the $Y$-axis is taken to be normal to the plane of incidence ( $X Z$ plane).

We use $\gamma$ and $\Gamma$ to denote the polarization angles of the input fundamental and output second harmonic beams, respectively. These angles are measured relative to the plane of incidence, where for example, $\gamma=s,(\Gamma=p)$ denotes the plane of polarization of the incident (harmonic) beam to be perpendicular (parallel) to the plane of incidence. We further abbreviate this polarisation notation for both the input and the output polarizations as $s$ for $(\gamma$ or $\Gamma=s)$, and $p$ for $(\gamma$ or $\Gamma=p)$.

Using equation (3) in the results of Bloembergen and Pershan for the case where the fundamental wavelength is much greater than $d,{ }^{11}$ the equations for the second harmonic field for media belonging to a $3 \mathrm{~m}$ symmetry are generated for a given polarization, (input, output) as:

$$
\begin{aligned}
& E_{p, p}^{2 \omega}=4 \pi i\left(\omega d c^{-1}\right)\left(a_{1}+a_{2} K_{1}\right)\left(E^{(\omega)}\right)^{2} \\
& E_{s, p}^{2 \omega}=4 \pi i\left(\omega d c^{-1}\right)\left(b_{1}+b_{2} K_{1}\right)\left(E^{(\omega)}\right)^{2} \\
& E_{s, s}^{2 \omega}=4 \pi i\left(\omega d c^{-1}\right)\left(c_{1} K_{2}\right)\left(E^{(\omega)}\right)^{2}
\end{aligned}
$$

where $a_{1}=\left(F_{z}^{2 \omega}\left(F_{x}^{\omega}\right)^{2} \chi_{z x x}+F_{z}^{2 \omega}\left(F_{z}^{\omega}\right)^{2} \chi_{z z z}+2 F_{x}^{2 \omega} F_{z}^{\omega} F_{x}^{\omega} \chi_{x z x}\right), a_{2}=F_{y}^{2 \omega}\left(F_{x}^{\omega}\right)^{2} \chi_{y y y}$, $b_{1}=F_{z}^{2 \omega}\left(F_{y}^{\omega}\right)^{2} \chi_{z x x}, b_{2}=-F_{y}^{2 \omega}\left(F_{y}^{\omega}\right)^{2} \chi_{y y y}$, and $c_{1}=F_{y}^{2 \omega}\left(F_{y}^{\omega}\right)^{2} \chi_{y y y}$. 
In these equations, $F_{j}^{\omega}$ and $F_{i}^{2 \omega}$ are the components of the linear and the second harmonic Fresnel factors within the middle layer. ${ }^{11,12}$ The values of these factors, which are in general complex, are not important for this paper and therefore they will remain masked within constants in equation (4-6) . Equations (4-6) can be generalised in a form which distinguishes the contribution of rotationally isotropic terms $(B)$ and rotationally anisotropic terms $(A)$ as

$$
E^{2 \omega}=B+A K(\varphi)
$$

$B$ is a linear combination of the isotropic components of the $\chi^{(2)}$ tensor: $\chi_{z x x}, \chi_{z z z}, \chi_{x z z}, A$ is proportional to the anisotropic susceptibility $\chi_{y y y}$ and $K(\varphi)$ denotes the sine and/or cosine variation of the azimuthal angle in equations (4-6).

The effect of the magnitude of $B$ relative to that of $A$ on the azimuthal variation of the SHG signal is known to affect the relative amplitudes and the number of peaks of the observed SHG signal as a function of the azimuthal angle due to the interaction between these terms ${ }^{13}$. With $B$ dominating, a 3-fold symmetry is expected from the SHG curves with a non-zero signal over the full azimuthal rotation. Reduction of $B$ should result in additional peaks appearing until the full six-fold symmetry is observed when $B$ is zero. The contribution of the $A$ and $B$ terms is easily controlled by the choice of the input and output polarisation angle. From equation (3), it can be seen that for $\Gamma=s, B$ is zero for any value of $\gamma$ hence the only term that should contributes to the observed SH signal is $A$, and hence, for $s, s$ (see equation (6)) a full six-fold symmetrical SHG curve is expected.

\section{EXPERIMENTAL}

A $532 \mathrm{~nm}$ Q-switched Nd:YAG frequency-doubled laser $(20 \mathrm{~Hz}$ repetition rate, $10 \mathrm{~ns}$ pulse width, pulse energy of $2 \mathrm{~mJ}$, Continuum, Minilite ) was used as the input beam. Figure 2 shows a schematic of the experimental set-up used for the rotation anisotropy measurements. 
The power of the input beam was controlled by rotation of the $\lambda / 2$ plate placed in the beam path between the laser head and the polarizing beam splitter (PBS1). The plane of polarization of the input beam was controlled by rotating the second $\lambda / 2$ plate. A beam splitter (BS) was used to direct a small fraction of the input beam into the photodiode (PD) for monitoring the power. The signal from the PD was also used to correct for any fluctuations in the SHG beam caused by the input beam instability. A $20 \mathrm{~cm}$ focal length lens (L1) was used to focus the beam to a spot size of $\sim 0.5 \mathrm{~mm}$ on the $z$-cut congruent $\mathrm{LiNbO}_{3}$ sample. $\mathrm{LiNbO}_{3}$ samples were cut from a wafer purchased from Crystal Technology, USA. A UV blocking filter (UVBF) was placed between $\mathrm{L} 1$ and the $\mathrm{LiNbO}_{3}$ to block any SHG signal generated along the optical train before the sample. The sample was held on a rotational stage which allowed the sample to rotate around its surface normal. The incident angle of the beam, $\theta$, was set to be $60^{\circ}$. A visible blocking filter (VBF) was placed in the path of the reflected beam to block any reflected part of the fundamental beam. The train of optics in both the input and the output paths of the beam was fixed on two separate movable arms which allowed variation of both the incidence and the reflection angles. The SHG signal was passed through a UV Rochon polarizer (PBS2) $p$ or $s$ polarization selction. An iris diaphram, A1, was placed after this polariser to block any diverted light. The signal was subsequently focused on the slit of a monochromator set to allow transmission of only $266 \mathrm{~nm}$ which was detected by the photomultiplier (PMT). After feeding the signal from the PMT into a preamplifier, the amplified signal and that from the PD were fed into a gate integrator before both were synchronously acquired and analyzed using a data acquisition unit (DAQ) and a personal computer (PC), triggered by the Q-switch signal from the laser.

The crystals used had a slab geometry for which both $z$ face surfaces were parallel. Due to the small thickness $(500 \mu \mathrm{m})$ and the spot size of the beam, multiple reflections occur between 
the two z-faces which results in a higher SHG signal being generated. The fundamental beam reflected from the bottom surface thereby generated a secondary SHG signal from the last few atomic layers as it emerged from the top surface. Due to multiple reflections, this contribution from the secondary SHG signal was larger than the primary SHG signal, as observed via RSHG. This secondary SH signal was minimised by roughening the bottom surface of the crystal with 600 grit sandpaper.

\section{RESULTS AND DISCUSSIONS}

A. Use of RSHG to reveal the symmetry of the interfacial layer of a z-cut $\mathrm{LiNbO}_{3}$ crystal

Table I summarizes the rotation anisotropy results on a $z$-cut $\mathrm{LiNbO}_{3}$ sample. The curves shown in Figure 3 to Figure 5 have the characteristics of a $3 \mathrm{~m}$ symmetry with symmetry repetition every $60^{\circ}$ or $120^{\circ}$ over the full $360^{\circ}$ rotation. Each point in these figures is an average of 250 accumulated measurements. The values in the curves are normalized to a maximum SHG efficiency value for $s, p$ shown in Figure 5. The same reasoning used to account for the bulk SHG null signal in a centrosymmetric medium can be used to put constraints on mirror planes with respect to the plane of incidence. In this case, the SHG signal should vanish when a mirror plane coincides with the plane of incidence for $s, s$ since both the input and output polarizations are perpendicular to the mirror plane. For a $3 \mathrm{~m}$ material, three mirror planes intersecting each other at $60^{\circ}$ are expected and are clearly represented by the minima in the SHG signal for $s, s$ in Figure 3. By the standard definition of the crystalline axes in $\mathrm{LiNbO}_{3},{ }^{14}$ the three equivalent $y$-axes lie along these mirror planes. With the mirror plane found in Figure 3, the $y$-axes locations are determined to an accuracy limited by the azimuthal angle steps chosen. Jung et al. ${ }^{9}$ have proposed and used $\mathrm{LiNbO}_{3}$ to demonstrate a simple method for the determination of crystalline axes of a non-linear uniaxial 
crystal via transmission SHG. The transmission geometry for SHG experiments has a few drawbacks compared with reflection SHG however. With reflection SHG, phase matching between the fundamental beam and the second harmonic beam is not required for sufficient SH signal to be generated and detected, hence making this method simpler.

It was shown in the theory part of this paper that the rotationally isotropic and anisotropic contributions to the measured SHG signal can be separated as $B$ and $A$, respectively. When $|A|<|B|$ the isotropic terms dominate and only three major peaks are observed. This result for the case of $p, p$ is shown in Figure 4 and is accounted for by the fact that $\chi_{z z z}$ for $\mathrm{LiNbO}_{3}$ is the largest coefficient compared to all other components of the $\chi^{(2)}$ tensor hence making the $B$ term the largest. With $s, p$ shown in Figure 5, the magnitude of the isotropic term is reduced compared to that of $p, p$ since there is no contribution from $\chi_{z z z}$ and $\chi_{x z x}$, and minor peaks (local maxima) subsequently appear. On fitting the curves for the $p, p$ and $s, p$ SHG results, a phase difference was introduced between $|A|$ and $|B|$ as discussed previously by Andersson $e t$ $a l .{ }^{10}$ The phase difference in this case accounts for the different phase contributions between the $\chi^{(2)}$ tensor components in the isotropic and the anisotropic terms in the observed $\mathrm{SH}$ signal. For $s, s$ the full six-fold symmetry is observed since only an anisotropic term is contributing in this case.

On fitting the curves, $\varphi$ in equations (4-6) was replaced by $\varphi+\varphi_{0}$ with $\varphi_{0}$ denoting the offset between the laboratory-fixed axis and the crystal-fixed axis as it was not possible to position the sample on the stage with the required degree of precision. From the curve fitting procedure however, the location of the $x$ and $y$-axes are thereby determined with the prior 
knowledge only of the optical axis. Table II shows the values of $\varphi_{0}$ determined from the curve fitting procedure.

B. Use of RSHG to detect the inversion of the $y$-axis $(+y \Leftrightarrow-y)$ on reversal of the spontaneous polarisation

The remaining part of the paper focuses on the use of RSHG to detect changes in the $y$-axis polarity caused by domain inversion along the $z$-axis. The standard sign convention for the $y$ and $z$ axes is related to (and defined by) the piezoelectric effect: compression of either $y$ or $z$ faces results in a negative voltage appearing on their positive faces. There is a direct relationship between the sign of these faces and the relative atomic arrangement order along these axes within the crystal. The atomic arrangement is of interest here since it directly influences any atomic polarization within the medium and hence any changes within this order can therefore be detected for example though the SHG technique. Within $\mathrm{LiNbO}_{3}$, the cation order along the $z$-axis from the $+z$ to the $-z$ face is $\mathrm{Nb}^{5+}, \mathrm{Li}^{+}, \mathrm{Vacancy}, \mathrm{Nb}^{5+}, \ldots$ as shown in Figure 6 (d). Along the $y$-axis, also shown in Figure 6 (d) there is also a corresponding direction-dependent order of cations, sandwiched between the two planes of oxygen ions forming octahedron complexes with the cations $\left(\mathrm{NbO}_{6}\right.$ and $\left.\mathrm{LiO}_{6}\right)$. The reversal of $\mathbf{P}_{\mathbf{s}}$ is a result of the forced relative displacement of the cations along the $z$-axis and in the case of $\mathrm{LiNbO}_{3}$, the cationic order reverses which also reverses the ordering along the $y$-axis as shown in Figure 6 (e). It is these latter changes along the $y$-axis that we wish to investigate here via RSHG.

The reversal of $\mathbf{P}_{\mathbf{s}}$ and hence the reversal of the $z$-axis within $\mathrm{LiNbO}_{3}$ can be pictured by the physical operation on a system of Cartesian coordinates with the same point group properties 
as $\mathrm{LiNbO}_{3}$ as shown in Figure 6 (a). In this figure, the $x$-axis is not explicitly shown since any effects on it upon inversion or rotation by $180^{\circ}$ around any axes $\left(\mathrm{C}_{2}\right.$ ( axis of rotation)) will be masked by the mirror plane that exists along the $y z$ plane. Therefore, inversion of all three $(\mathrm{x}, \mathrm{y}, \mathrm{z})$ crystal axes can also be achieved by $\mathrm{C}_{2}(x)$ on Figure 6 (a). From Figure 6 (a), the reversal of the $z$-axis can be achieved in several ways, either by rotating the $\operatorname{crystal} \mathrm{C}_{2}(y)$, as shown in Figure 6 (b), or by inversion of the whole crystal relative to the laboratory-fixed axes as shown in Figure 6 (c). All these operations result in the reversal of the $z$-axis $(+z \Leftrightarrow-z)$ but as shown in Figure $6(\mathrm{~b}), \mathrm{C}_{2}(y)$ on Figure 6 (a) leaves the direction of the $y$ axis unchanged. Alhough this $\mathrm{C}_{2}(y)$ operation on Figure 6 (a) results in $+z \Leftrightarrow-z$, it cannot lead to the physical domain inversion along the $z$-axis since it would leave the sign of the $y$ axis unchanged and hence leaving the order of cations along the $y$-axis intact. Domain reversal is therefore equivalent to the operation shown in, Figure 6 (c), in which both the $y$ and $z$ axes are reversed $(+y \Leftrightarrow-y,+z \Leftrightarrow-z$ ) within the poled area, which is equivalent to the inversion of the whole crystal or just the $\mathrm{C}_{2}(x)$ on Figure 6 (a) for a crystal with the mirror planes along the $y z$ plane.

The information about the polarity of the $y$-axes cannot be determined from the $s, s$ curves, which shows the location of the mirror planes and hence of the $y$-axis with respect to the incidence plane by the minimum SHG signal, and therefore other polarization combinations must be used. With $\Gamma=\mathrm{p}$, the interaction between the rotationally isotropic and anisotropic components of the $\chi^{(2)}$ tensor should reveal the polarity of the crystalline $y$ - and $z$-axes relative to the input and output geometry. For $s, p$ shown in Figure 5, the location of the global and local maxima coincide with the minima for $s, s$ and hence yield information about the polarity of the $y$ direction. 
The relationship between the sign of the $y$ and $z$ axes with respect to the plane of incidence was investigated using rotation anisotropy RSHG experiments on a virgin $\mathrm{LiNbO}_{3}$ sample with known crystalline axes and polarities. Though the three $y$-axes are identical, they will contribute to the observed SHG anisotropy at different azimuthal angles. We therefore argue below from the SHG results, that it is crucial to label these three equivalent $y$-axes in order to detect any polarity changes after $z$-axis inversion. During the experiment, the positions of the $y$-axes were known for any azimuthal angle.

Table III shows the summary of the results on both faces of the virgin $\mathrm{LiNbO}_{3}$ for $s, p$. The direction of rotation around the $z$-axis on the $+z$ and $-z$ faces was clockwise and anticlockwise as shown by Figure 7(a) and Figure 8 (a), respectively. The reversal of the $z-$ axis was achieved through $C_{2}(x)$ such that $+y_{1} \Leftrightarrow-y_{1},+y_{2} \Leftrightarrow-y_{3},+y_{3} \Leftrightarrow-y_{2}$ and $+z \Leftrightarrow-z$ while the sign of the $x$-axis was left unchanged relative to the plane of incidence, as shown by the previous figures. From Table III, it is then straightforward to make the following conclusions for $s, p$ : On the $+z$ face, the $+y$ direction gives a global maximum for the SHG signal at $\varphi=30^{\circ}$, as shown in Figure 7(b), under rotation anisotropy when the $+y$ directions are aligned parallel and pointing along the positive direction of the $X$-axis (referred to as $+X$ in Table III). On the $-z$ face, the $-y$ direction gives a global maximum for the SHG signal at $\varphi=90^{\circ}$ as shown in Figure 8 (b), from rotation anisotropy when the $-y$ directions are parallel and pointing along the positive direction of the $X$-axis.

It is clear from the labelled y-axes in Figure 8 (a) and the summary in Table III that rotating the crystal clockwise while probing the $-z$ face will results in the same SHG curve, Figure 9 (b), as the one in Figure 7 (b) where the experiments were performed on the $+\mathrm{z}$ face. 
However, the contributing y-axes for global maxima, as an example, in these two cases possess opposite signs. The effect of the direction of rotation on the same crystal face for these SHG experiments is to shift the azimuthal angle by $180^{\circ}$, therefore, Figure 9 (b) is just the continuation of Figure 8 (b) from $\varphi=180^{\circ}$ onward. Thus, performing rotation anisotropy SHG experiments with the same sense of rotation on different faces must result in similar curves.

The above conclusions were then used to detect changes of the sign of the $y$-axis imposed by the reversal of $\mathbf{P}_{\mathbf{s}}$. The inset diagram in Figure 10 shows a schematic top-view of a $\mathrm{LiNbO}_{3}$ sample that has had half its area domain inverted. Where, the $+z$ face of the virgin part is pointing out of the page and that of the domain inverted (- $z$ face $)$ is pointing into the page. RSHG rotation anisotropy experiments were performed for $s, p$ on the virgin part and the crystal was then translated along the $+X$ direction and the same experiment was repeated on the poled area with the same rotation direction. The results are shown in Figure 10 and their clear similarity show conclusively that the sign of the $y$-axes must have been reversed in the poled area $(-z$ face $)$ relative to that in the virgin area $(+z$ face $)$ since similar curves are observed from both the $-z$ and $+z$ faces. These results show conclusively the use for RSHG for the detection of the reversal of the $y$-axis as a result of the domain reversal procedure.

\section{CONCLUSIONS}

RSHG experiments were used to reveal the interfacial layer symmetry and determine the crystallographic axes of a $z$-cut congruent lithium niobate crystal. The high UV absorption within this material has been used effectively to limit the generation of the SHG signal to the interfacial layer and hence extending the use of RSHG to the non-centrosymmetric materials. Based on the absorption depth of the medium, careful choice of the fundamental wavelength 
and hence variation of the interfacial layer thickness should result in the approach used in this paper being sensitive to a monolayer. As a special case, RSHG was also used to detect the reversal of the $y$-axis caused by the domain inversion along the $z$-axis. This effect is made possible by the interaction between the isotropic and anisotropic second-order susceptibilities and hence it is dependent on the input and output polarization angles. 


\section{ACKNOWLEDGEMENTS}

T. J. S gratefully acknowledges a Commonwealth Scholarship to the United Kingdom. 


\section{References:}

1 P. N. Butcher and D. Cotter, The element of Nonlinear optics (Cambridge Univesity Press, Cambridge, 1990).

2 D. L. Mills, Nonlinear Optics, Basic Concepts (Springer, Berlin, 1998).

3 T. Stehlin, M. Feller, P. Guyot-Sionnest, and Y. R. Shen, Opt. Lett. 13, 389 (1988).

4 A. M. Mamedov, Opt. Spectrosc. 56, 645 (1984).

5 E. Wiesendanger and G. Guntherrodt, Solid State Commun. 14, 303 (1974).

$6 \quad$ Y. R. Shen, Appl. Phys. B 68, 295 (1999).

7 D. A. Kleinman, Phys. Rev. 126, 1977 (1962).

8 L. Reining, R. D. Sole, M. Cini, and J. G. Ping, Phys. Rev. B: Condens. Matter 50, 8411 (1994).

9 C. Jung, B. K. Rhee, and D. Kim, Appl. Opt. 39, 5142 (2000).

10 S. K. Andersson, M. C. Schanne-Klein, and F. Hache, Phys. Rev. B: Condens. Matter 59, 3210 (1999).

11 N. Bloembergen and P. S. Pershan, Phys. Rev. 128, 606 (1962).

12 T. G. Zhang, C. H. Zhang, and G. K. Wong, J. Opt. Soc. Am. B: Opt. Phys. 7, 902 (1990).

H. W. K. Tom, T. F. Heinz, and Y. R. Shen, Phys. Rev. B: Condens. Matter 51, 1983 (1983).

R. S. Weis and T. K.Gaylord, Appl. Phys. A 37, 191 (1985). 


\section{Figure captions}

Figure 1 Crystal-fixed axes $(\mathrm{x}, \mathrm{y}, \mathrm{z})$ relative to the laboratory-fixed axes $(\mathrm{X}, \mathrm{Y}, \mathrm{Z}) . \theta$ and $\varphi$ are the incident and the azimuthal angles: $\mathrm{p}$ and $\mathrm{s}$ are the polarization of the beam as described in the text.

Figure 2 Experimental set-up. The dotted line shows the electrical signal path. PBS1 and PBS2 are the input and output polarizer, BS: beam splitter. PD: the photodiode. UVBF and VBF are the UV and visible blocking filters. L1, L2 and L3 are lenses. A1: iris diaphragm. PMT: Photomultiplier tube. DAQ: data acquisition unit. PC: personal computer.

Figure 3 The triangles show experimental data for $s, s$ while the solid line is the fitted curve.

The error bars shows a $1 \sigma$ standard error. $\theta=60^{\circ}$.

Figure 4 The open circles show experimental data with $p, p$ while the solid line is the fitted curve. The error bars shows a $1 \sigma$ standard error. $\theta=60^{\circ}$.

Figure 5 The open squares show experimental data with $s, p$ while the line is the fitted curve.

The error bars shows a $1 \sigma$ standard error. $\theta=60^{\circ}$.

Figure 6 Possible physical operations towards inversion of a system with mirror planes along the yz plane. (a) and (d) are the coordinate system and the $\mathrm{LiNbO}_{3}$ atomic arrangement in a virgin state. (b) Shows the coordinates system rotated by $180^{\circ}$ around $y$-axis $\left(\mathrm{C}_{2}(y)\right)$ from the virgin state. (c) Shows the coordinates system rotated by $180^{\circ}$ rotation around $x$-axis $\left(\mathrm{C}_{2}(x)\right)$, which is similar to the inversion of the whole crystal. (e) $\mathrm{LiNbO}_{3}$ atomic arrangement in a domain inverted.

Figure 7 (a) The labelled $y$-axes with the $+z$ direction pointing out of the page and also showing the direction of rotation. (b) RSHG curves with the global maximum value ( $\varphi$ $=30^{\circ}$ ) corresponding to the alignment of the $+y_{3}$ direction along the $+X$ direction. 
The SHG signal was normalized to the value at $\left(\varphi=30^{\circ}\right)$ and the error bar are calculated as in the previous figures in this paper.

Figure 8 (a) The labelled y-axes with the $-z$ direction pointing out of the page (b) RSHG curves with the local maximum value $\left(\varphi=30^{\circ}\right)$ corresponding to the alignment of the $+y_{3}$ direction along the $+X$ direction. The SHG signal was normalized to the value at $\left(\varphi=90^{\circ}\right)$ and the error bar are calculated as in the previous figures in this paper.

Figure 9 (a) The labelled $y$-axes with the $-z$ direction pointing out of the page. (b) RSHG curves with the global maximum value $\left(\varphi=30^{\circ}\right)$ corresponding to the alignment of the $-y_{2}$ direction along the $+X$ direction. The SHG signal was normalized to the value at $\left(\varphi=30^{\circ}\right)$ and the error bars are calculated as in the previous figures in this paper.

Figure 10 Inset diagram shows the schematic top-view of a half re-poled $\mathrm{LiNbO}_{3}$ sample. SHG results from the $+z$ face of the virgin part (filled circles) and $-z$ face of the poled area (open circles). The SHG signal was normalized to the value at $\left(\varphi=90^{\circ}\right)$ and the error bars are calculated as in the previous figures in this paper. 
Figure 1

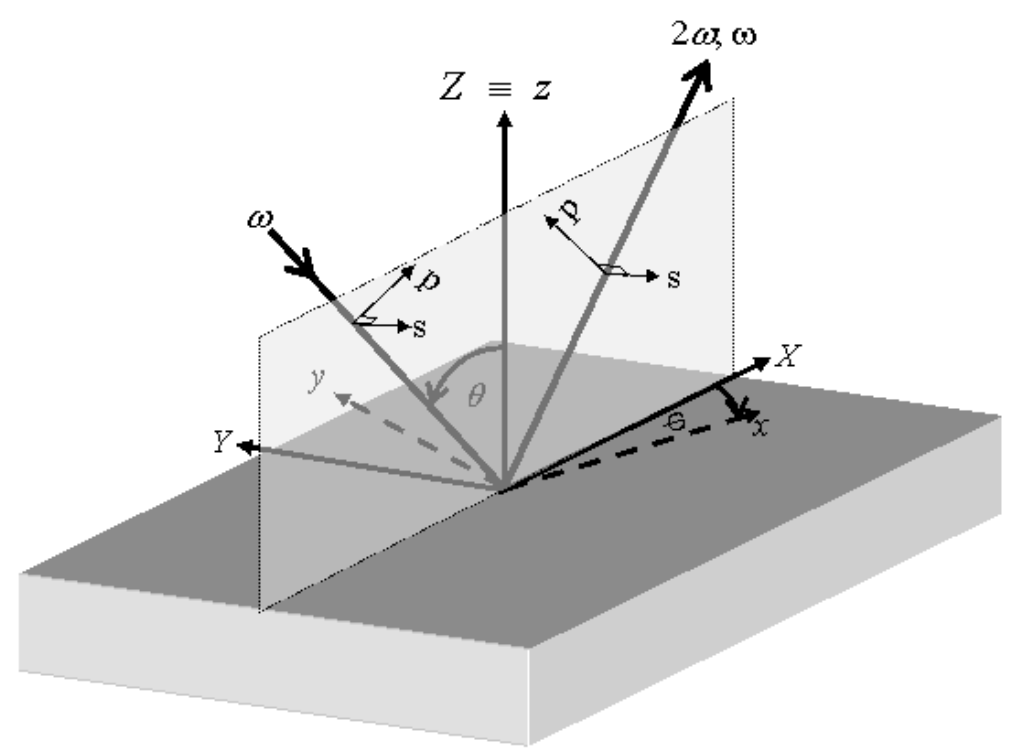


Figure 2

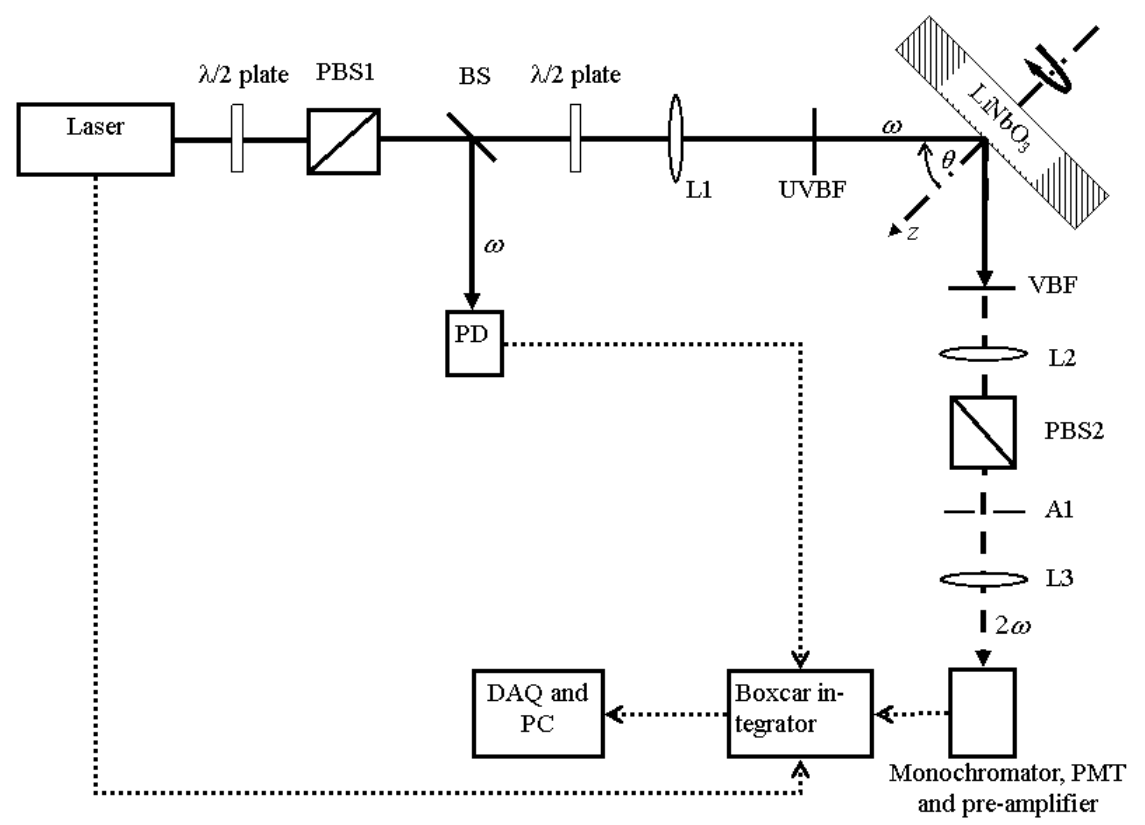


Figure 3

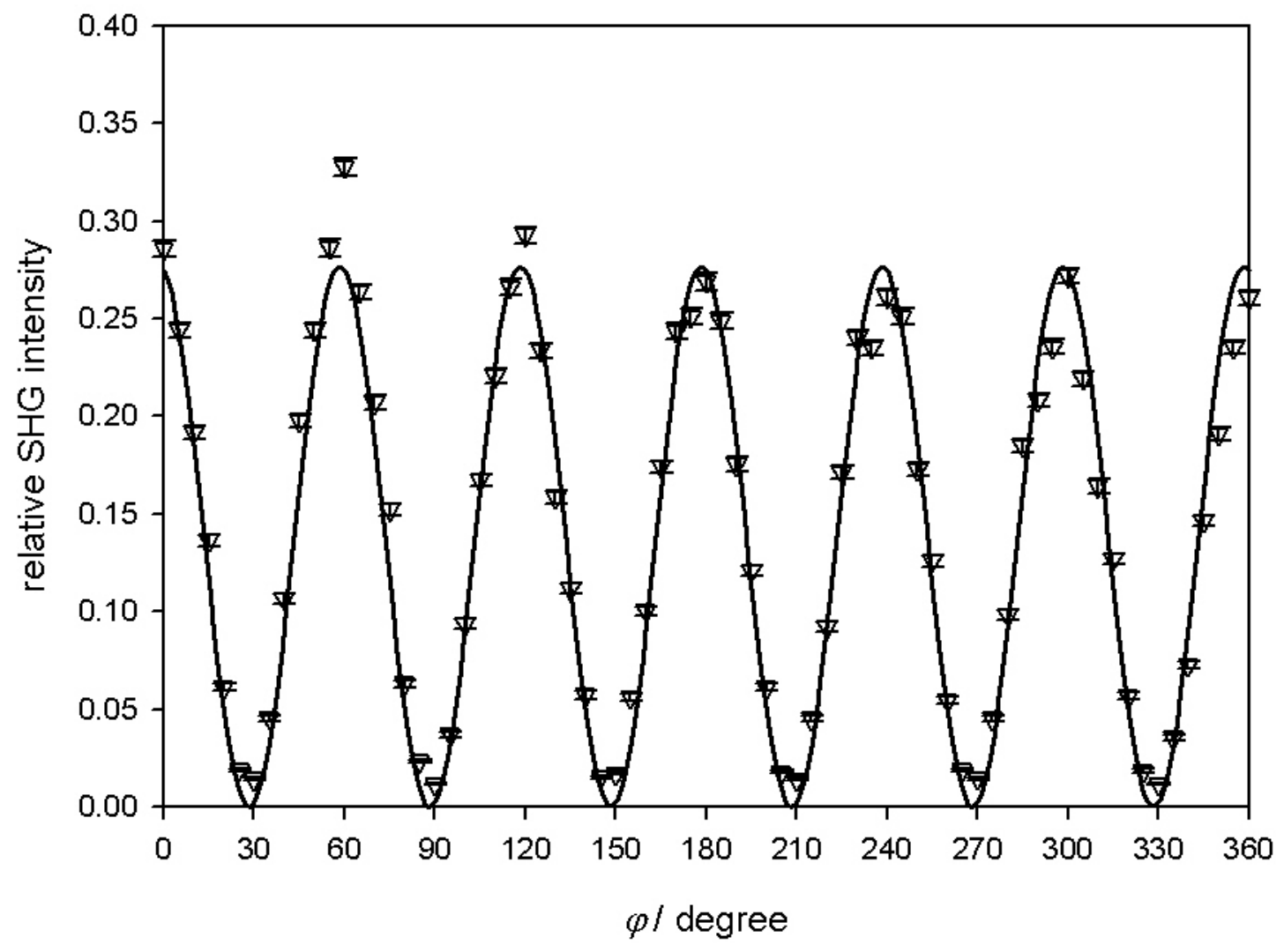


Figure 4

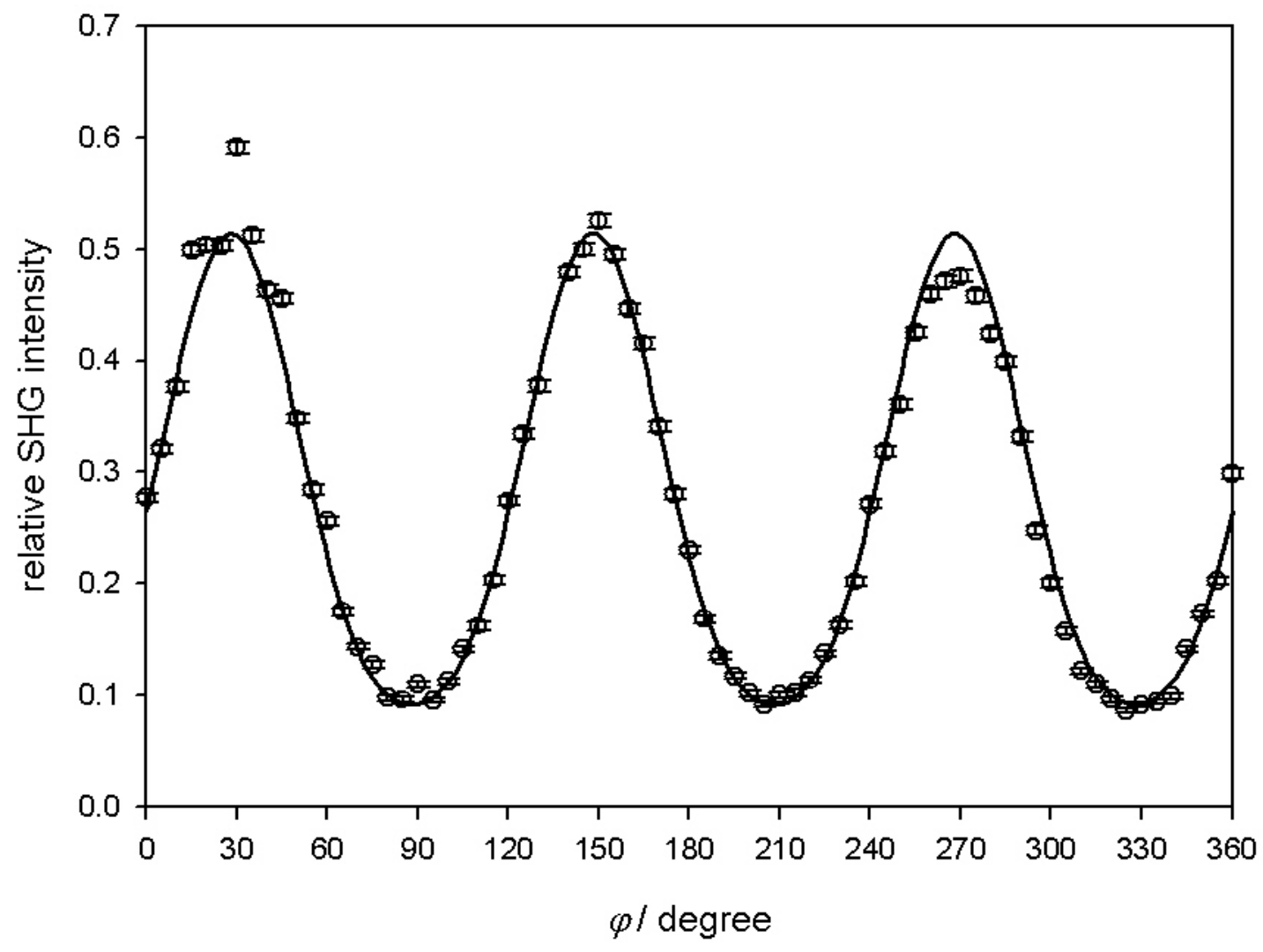


Figure 5

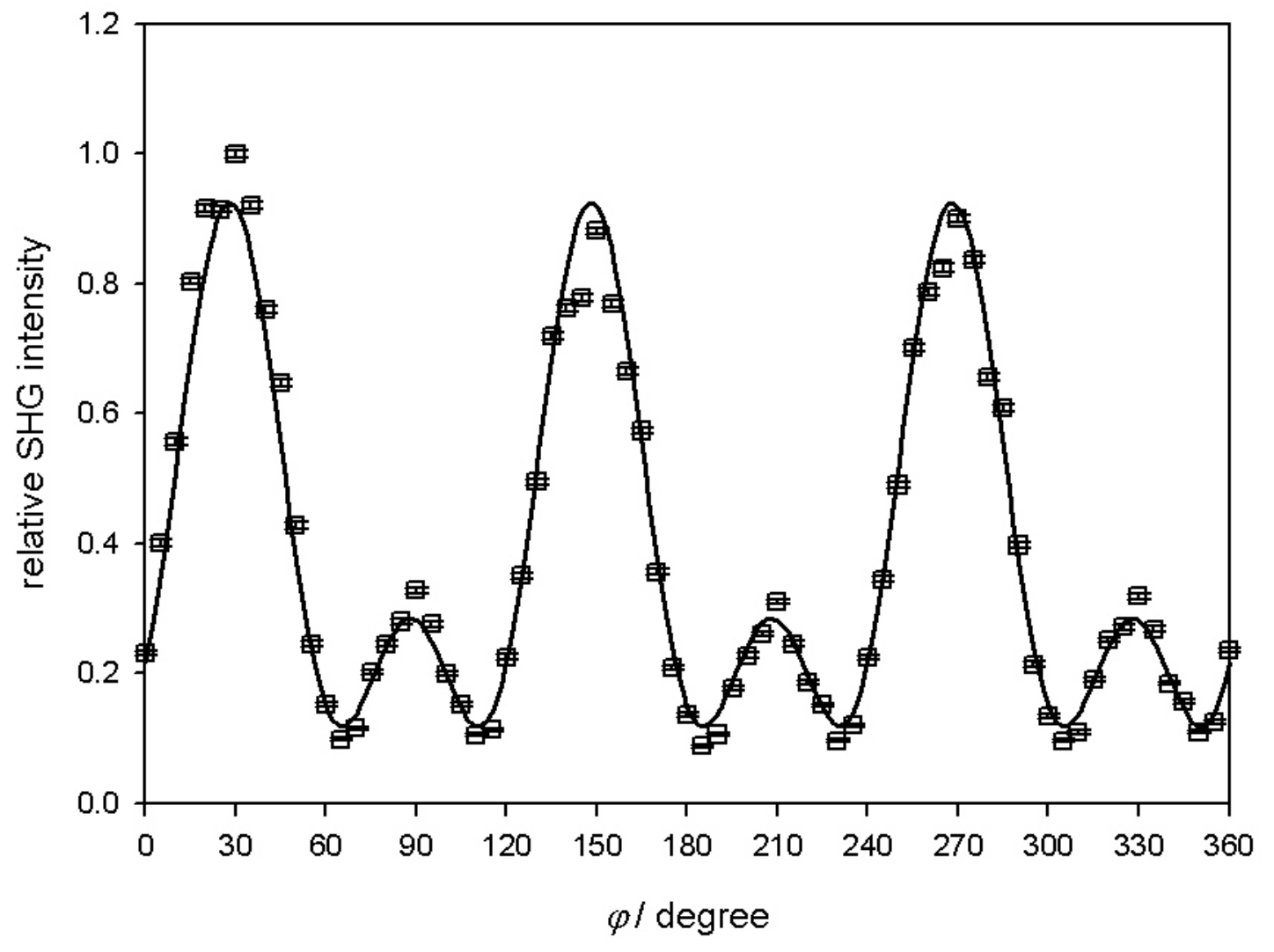


Figure 6

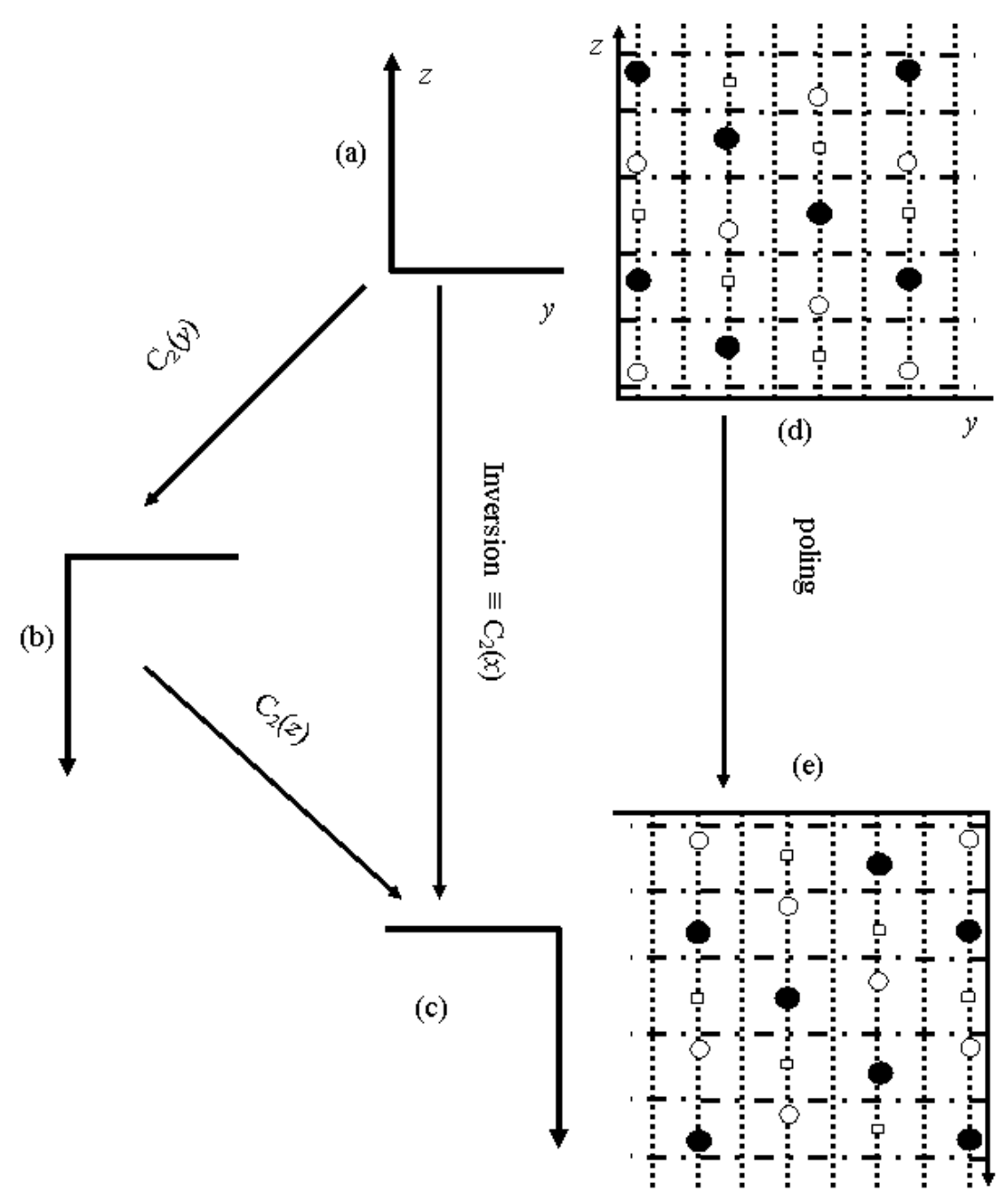

$\bigcirc \mathrm{Nb}^{5+} \quad \bigcirc \quad \mathrm{Li}^{+} \quad \square \quad$ Vacancy

- - - . Oxygen plane along the xy-plane

Oxygen plane along the xz-plane 
Figure 7

(a)

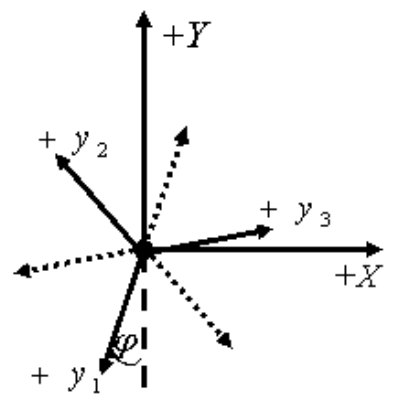

(b)

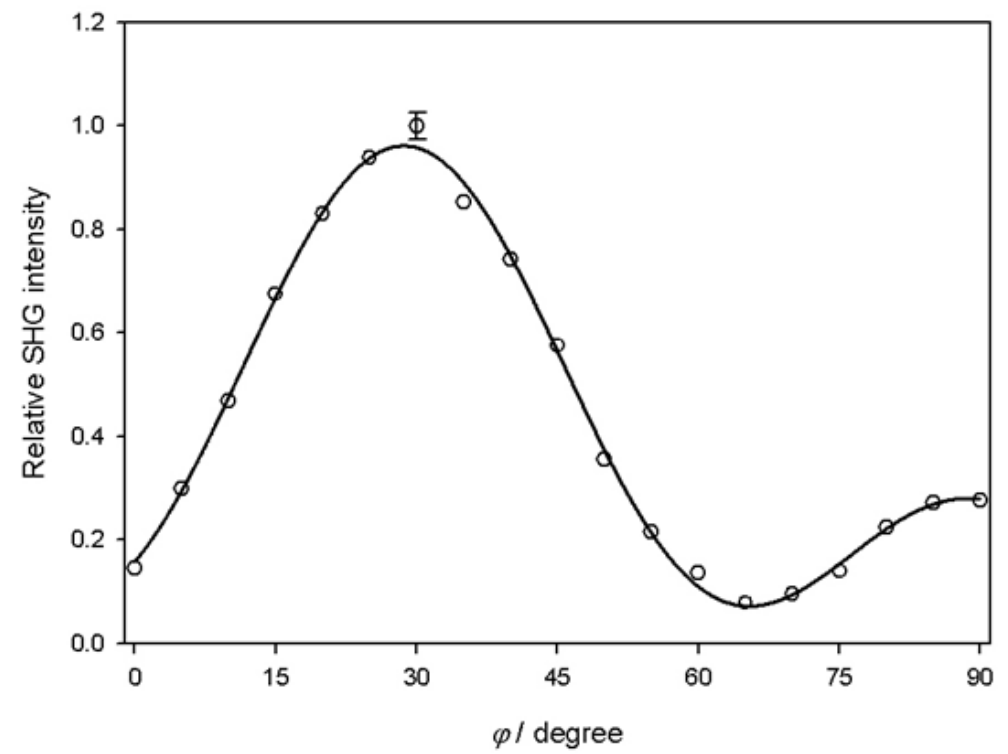


Figure 8

(a)

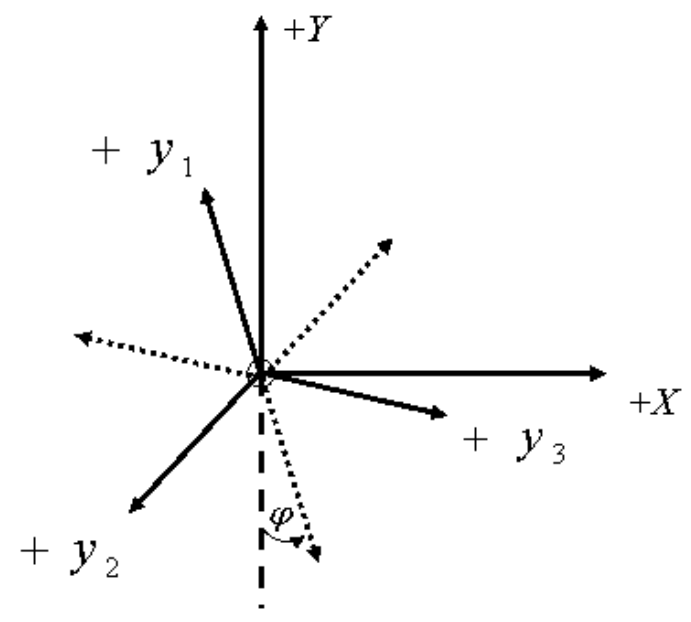

(b)

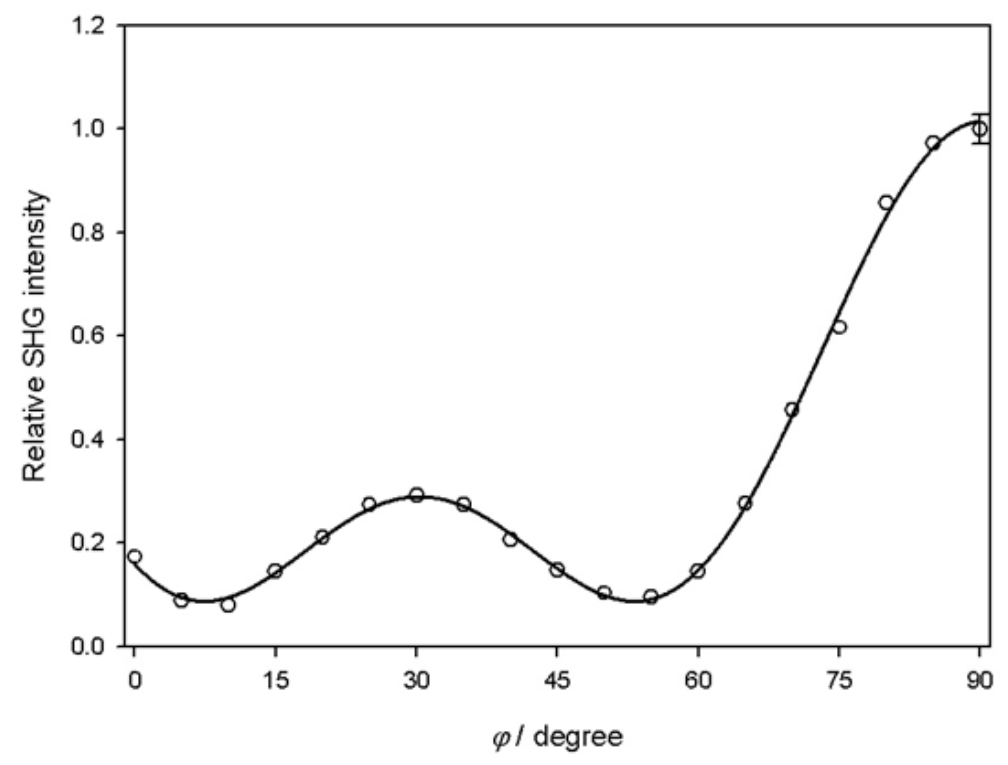


Figure 9

(a)

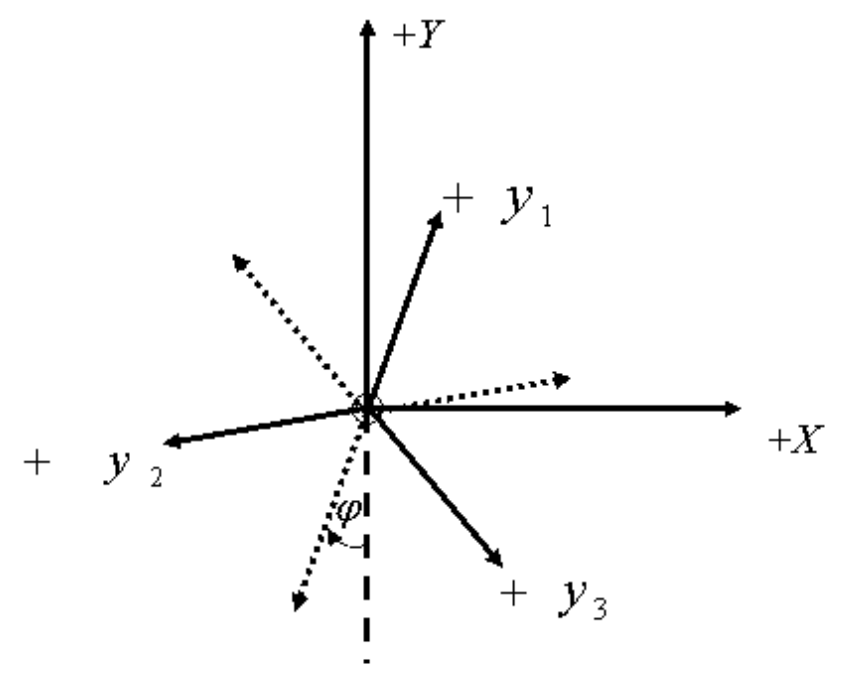

(b)

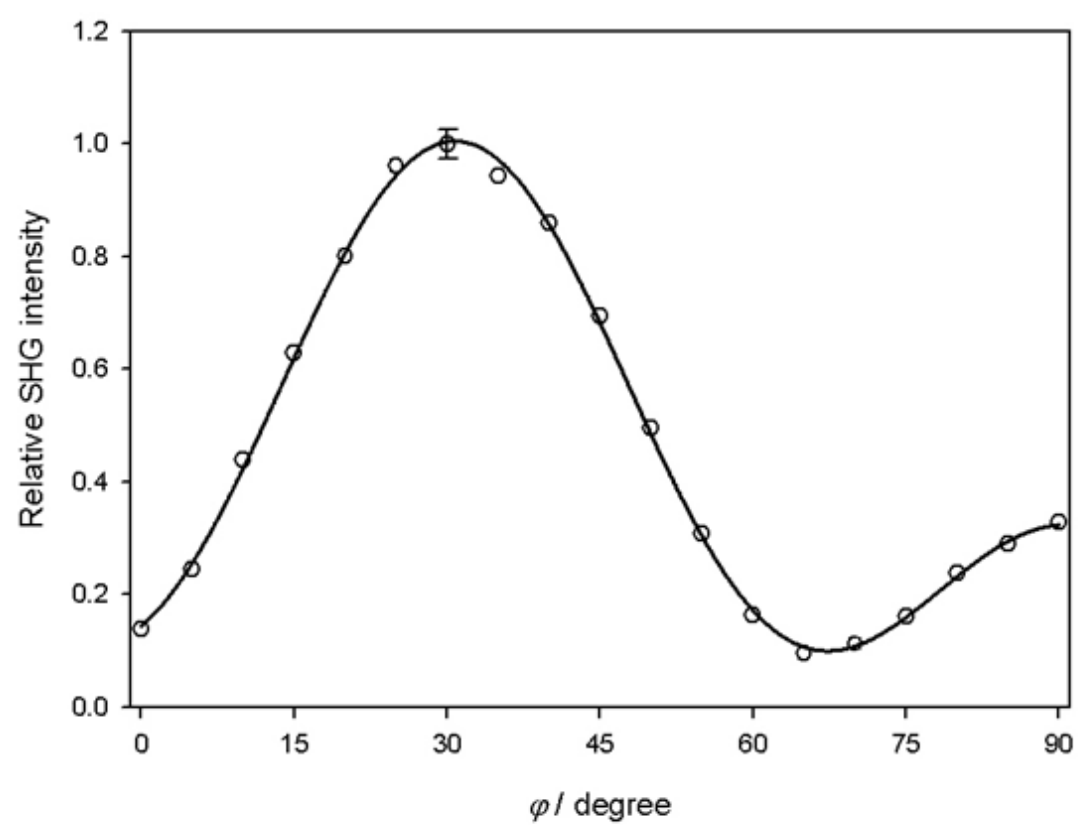


Figure 10

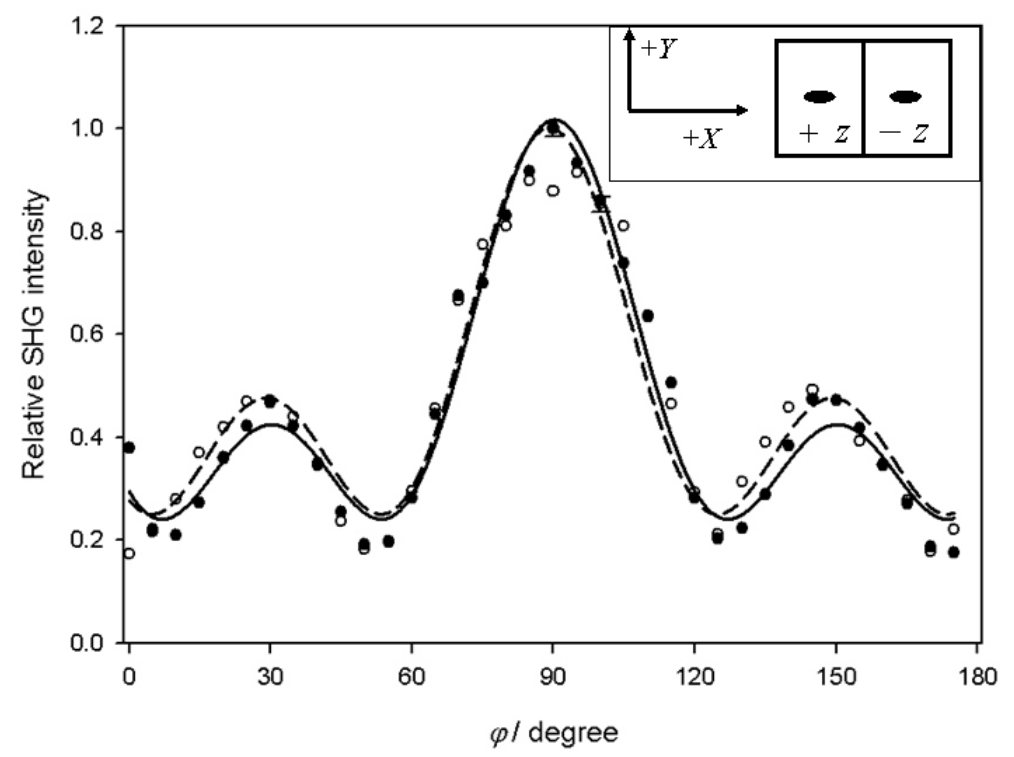


Table I. Summary of the rotational anisotropy results.

\begin{tabular}{|llll|}
\hline$\gamma$ & $\Gamma$ & Terminology & Figures \\
\hline$s$ & $s$ & $s, s$ & Figure $\mathbf{3}$ \\
$p$ & $p$ & $p, p$ & Figure $\mathbf{4}$ \\
$s$ & $p$ & $s, p$ & Figure $\mathbf{5}$ \\
\hline
\end{tabular}


Table II. Values of $\varphi_{o}$ determined from the curve fitting procedure.

\begin{tabular}{|llll|}
\hline & $p, p$ & $s, p$ & $s, s$ \\
\hline$\varphi_{0} /$ degree & $-1.9+/-0.3$ & $-2.0+/-0.2$ & $-1.6+/-0.2$ \\
\hline
\end{tabular}


Table III. Summary of the RSHG results for both faces of the virgin LiNbO3 for $s, p$.

\begin{tabular}{|lllll|}
\hline Crystal face & $\varphi=0^{\circ}$ & Global maxima & Local maxima & Figure \\
\hline$+z$ & $+y_{1}$ along $-Y$ & $+y_{3}$ along $+X$ & $-y_{1}$ along $+X$ & Figure 7 \\
$-z$ & $-y_{1}$ along $-Y$ & $-y_{1}$ along $+X$ & $+y_{3}$ along $+X$ & Figure 8 \\
$-z$ & $-y_{1}$ along $-Y$ & $-y_{2}$ along $+X$ & $+y_{1}$ along $+X$ & Figure 9 \\
& & & & \\
\hline
\end{tabular}

